\title{
Toward Fashion-Brand Recommendation Systems Using Deep-Learning: Preliminary Analysis
}

\author{
Yuka Wakita, Kenta Oku, and Kyoji Kawagoe
}

\begin{abstract}
Recently, the number of Electronic Commerce users has been rapidly increasing with the spread of the Internet. However, users cannot easily find their preferred clothes items among the enormous number on the Internet. As a method for solving this problem, we propose a fashion-brand recommendation system using a deep learning method. This system increases the likelihood that a user will find his/her favorite clothes items. The user must first determine his/her favorite fashion-brands. In this paper, we evaluate the effectiveness of using a deep learning method in a fashion-brand recommendation system. The preliminary analysis shows that the fashion-brand recommendation method using deep learning can dramatically improve the recommendation accuracy as compared with other machine learning methods.
\end{abstract}

Index Terms - Deep learning, fashion, recommend.

\section{INTRODUCTION}

Recently, the number of Electronic Commerce (EC) users has been rapidly increasing with the spread of the WWW [1]. In particular, in Japan many manufacturers of fashion clothes and accessories have increased the sales volume of their products via EC sites. However, from an EC user's viewpoint it is difficult to search clothing and find the favorite item in which he/she is interested from the enormous amount of clothes offered on EC sites. Thus, many EC sites and various companies are developing a clothes item recommendation service to increase their revenue. This service can help the user find the items of clothing that they wish to purchase.

However, the results of a survey conducted by Nakamura $e t$ al. [2] showed that $56.7 \%$ of EC users had experienced failure in online shopping. As a reason for this failure, $49.2 \%$ of the subjects gave "I felt I had purchased clothes that were cheaper than I had expected," $47.4 \%$ gave "The clothes I purchased didn't fit me as well as I thought they would," and $41.5 \%$ gave "I felt that the fabric of the clothes was not as I had thought it would be." In contrast to these answers to the survey questions about on-line shopping failures, to those about in-store shopping failures, $44.9 \%$ of the subjects answered "I made impulse buys" and 34.3\% answered "I felt that I didn't look as well in the clothes at home as I did in the shop." The reason for the EC users' responses is that they can view the images of clothes items only via the browser and it is difficult for them to find the images of the actual items of clothing.

Moreover, more than 10 items are recommended to a user, and he/she has no time to check them all by searching images

Manuscript received January 12, 2016; revised March 21, 2016.

The authors are with Ritsumeikan University, Kusatsu-shi, Shiga, 525-0058, Japan (e-mail: is0148xe@ed.ritsumei.ac.jp, oku@fc.ritsumei.ac.jp, kawagoe@is.ritsumei.ac.jp). on the browser.

In order to solve this problem related to clothes item recommendation, we propose fashion-brand rather than item recommendation. Fashion-brand recommendation has two advantages. First, the user is easily able to imagine the brand items by understanding the brand concept, which is an important component of any fashion brand. The brand concept includes realistic settings, such as the age group, price range, and unique character of the brand, which allows target user segmentation. Taking into account the brand world, the brand concept also includes attributes such as the sewing methods, fabrics, and design. If users search clothes items according to their favorite brand, the likelihood that they will easily discover their favorite clothes items increases. Moreover, in the fashion brand search, the user can see multiple brand clothes items displayed. Users can acquire brand features from the plurality of a brand's clothes items and they can imagine an item of the brand using these brand features. In other words, the clothes items along a brand concept can help the user easily imagine an item of clothing, which leads to the prevention of purchase failure in online-shopping. Second, the fashion-brand recommendation system can appropriately reduce the number of the clothes items the user needs to check on the browser. The user needs to view only a few different types of clothes items after receiving the recommendation.

In recent years, deep learning methods have achieved an overwhelming accuracy improvement in a variety of fields, in particular, in image recognition and video processing. However, deep learning methods have been applied in only a few application areas of information recommendation. In general, to perform information recommendation, various recommendation methods have been proposed [3]. The methods can be categorized into two filtering techniques: collaborative and content-based. In the collaborative filtering technique, items are recommended by using the item evaluation information of other users, whereas in the content-based filtering technique, items are recommended that are similar to the known item according to the content feature similarity. These two techniques can easily be unified to attain recommendations that are more accurate than those attained by applying conventional recommendation methods.

In this paper, we propose a fashion-brand recommendation method that uses a deep learning technique to obtain higher recommendation accuracy. The method is tailored to the preferences of the user who utilizes the fashion-brand classifier model. We evaluated the accuracy of the method by using a dataset consisting of users' favorite items that appeared in user profiles acquired from actual fashion Social Network Sites (SNSs). We compared our method with the 
traditional machine-learning methods: support vector machine (SVM) and random forest (RF).

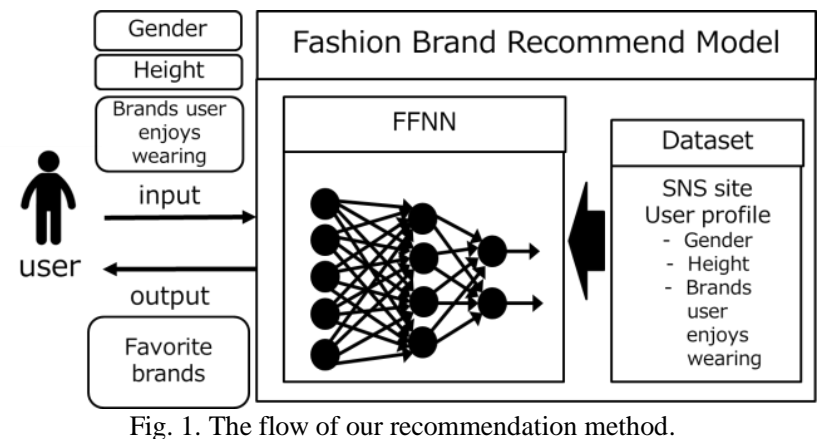

Recently, the number of Electronic Commerce users has been rapidly increasing with the spread of the Internet. However, users cannot easily find their preferred cloth items among the enormous number on the Internet.

\section{PRevious Fashion-Brand ReCOMmendation Method}

We previously proposed a fashion-brand recommender system using brand association rules and features [4]. In this system, fashion brands having a style similar to the user's favorite brands are recommended to the user. The system was realized by using association rules acquired from the user-brand matrix. However, one crucial disadvantage of this method is that it cannot recommend a fashion brand to a user if the user has no favorite fashion brands.

\section{PROPOSED FASHION-BRAND RECOMMENDATION METHOD}

In this paper, we propose a fashion-brand recommendation method that uses deep learning. Fig. 1 shows the flow of our recommendation method. First, a user inputs "gender," "height," and "brands the user enjoys wearing." Then, our fashion-brand recommendation model predicts the favorite brands of the user and directs the user to the predicted favorite brands. We constructed our recommendation model using deep learning.

\section{A. Data Set Used for Pre-learning}

The data set to be learned is a profile item on the well-known fashion SNS "WEAR" in Japan. The data set includes the items "gender," "height," "brands the user enjoys wearing," and "favorite brands." It uses these four items in the dataset for learning. Three of these items are the same as the user-input items shown in Fig. 1. In addition, we used these three items as input features in the training data set for learning, after considering the following.

\section{1) Gender}

A fashion brand has a target layer, for example, women's or men's clothing shops.

\section{2) Height}

For clothing, it is important to understand the user's body shape. "Do you know a fashion brand that suits short people?" and "Please recommend a fashion brand that suits tall people" are frequent comments on opinion exchange sites and in fashion magazines. Therefore, we consider it possible to represent the fashion brand features by using "height."

\section{3) Brands user enjoys wearing}

"Brands user enjoys wearing" and "favorite brands" are considered to be the same. However, this is not necessarily true for the following reasons. A user may possess clothes that another person bought for him/her; as when purchasing daily necessities, people do not buy clothes only in a vicinity where the choice of shops is unlimited; a person may enjoy wearing a garment, not because it is a favorite brand, but because it is practical; and when shopping for clothes, people consider the clothes they already possess.

Next, we normalized the data to the minimum required in order to learn this data set on the recommender model. We describe the details of each normalization in Table I.

TABLE I: NoRmaLIZATION OF THE INPUT DATA SET ITEMS To BE APPLIED

\begin{tabular}{|c|c|c|c|}
\multicolumn{4}{|c|}{ To TRAINING } \\
\hline \hline gender & Dimensions & Value range & Example \\
\hline \hline "female" $=0$ \\
height
\end{tabular}
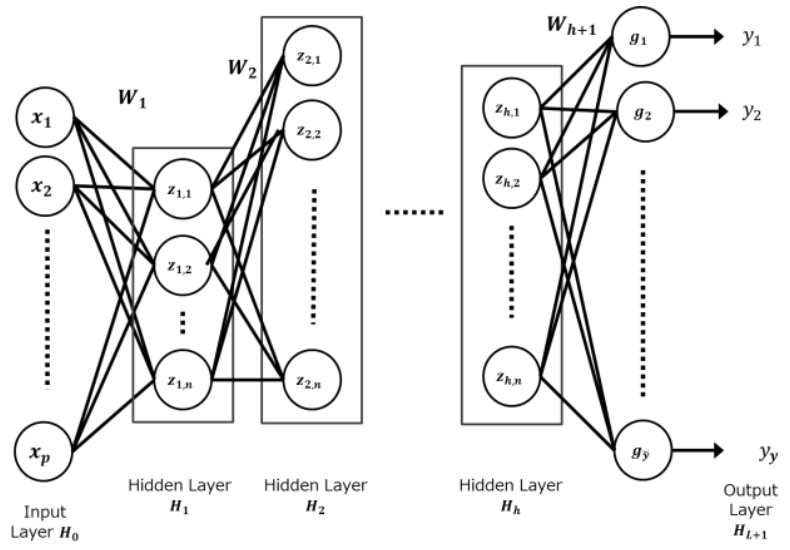

Fig. 2. The structure of the FFNN model.

The gender item has the two values: male and female. Females are represented by 0 , males by 1 . The unit of the height item is $\mathrm{cm}$. We divided the numerical values of the height item by $200 \mathrm{~cm}$ in order to represent this item by a range of 0-1. "Brands user enjoys wearing" has a plurality of brands. Each vector-item has a value of zero or one. The dimensionality is the same as the number of brands in the data set. If a user indicates a brand as one of the usual brands, the vector-item corresponding to the brand is set to one, otherwise the item is set to zero. Although the output, "favorite brand" that were extracted from the site originally is a list of brands, we choose the most favorite brand among the brands. It is because the learning task and the recommended brand should be simple as possible. The use of the whole favorite brand list in the learning task remains as our future work. 


\section{B. Deep Learning}

The deep learning network used in the recommendation method is the well-known feed forward neural network (FFNN) [5]. Fig. 2 shows the structure of the FFNN model used. By setting the parameters, it is possible to build different models.

$x$ : input features

$y$ : output features

$p$ : total number of input values expected

$h$ : total number of hidden layers

$n$ : number of corresponding layer neurons

$o$ : total number of output values expected

$z$ : activation function of the hidden layer

$g$ : activation function of the output layer

$L$ : loss function

The loss function is used to estimate the weight, $\mathbf{W}$, set for each layer, it is necessary to set this function.

\section{PRELIMINARY EXPERIMENT}

The purpose of the preliminary experiment was to verify that deep learning yields a higher prediction accuracy than other, conventional, machine learning methods. The results of the preliminary experiments for three methods, deep learning, SVM, and RF, were compared. For each approach, the default settings without tuning were used.

\section{A. Data Set Used in the Preliminary Experiment}

The data set used in the preliminary experiments consists of the profile item of the well-known fashion SNS "WEAR" in Japan, acquired on September 27, 2015. The data set is composed of 115 people profile items. We conducted a normalization of the data set using the method proposed in Section III. Table II shows the details of the normalized data set that was used. Fig. 3 shows the first 10 of data set as an example of our data set.

TABLE II: NormalizATION of the InPut Data Set Items To Be APPLIED TO TRAINING

\begin{tabular}{|c|c|}
\hline \hline The total number of the data set & 419 \\
\hline The total number of "Brands user wear well" & 124 \\
\hline The total number of the "favorite brands" & 10 \\
\hline The total number of the dimensions & 126 \\
\hline \hline
\end{tabular}

\section{B. Parameters Used in Deep Learning}

The fashion brand recommendation model was created using the "H2O" library. Table III describes the configuration parameters for building the fashion recommender model.

TABLE III: PARAMETERS USED IN DEEP LEARNING

\begin{tabular}{|c|c|c|}
\multicolumn{3}{|c|}{ TABLE III: PARAMETERS USED IN DEEP LEARNING } \\
\hline & input features & value \\
\hline$x$ & output features & 126 \\
\hline$y$ & total number of input values expected & 1 \\
\hline$p$ & the total number of hidden layer & 126 \\
\hline$h$ & the number of the corresponding layer neurons & 200 \\
\hline$n$ & the total number of output value expected & 10 \\
\hline$o$ & activation function of the hidden layer & Rectified \\
& & Liner \\
\hline$z$ & activation function of the output layer & Softmax \\
\hline$g$ & Loss function & Cross \\
& & Entropy \\
\hline$L$ & & \\
\hline \hline
\end{tabular}

\section{The Execution Details on the Three Methods}

This experiment was performed using the $\mathrm{R}$ language. $\mathrm{R}$ libraries of h2o, e1071 and random Forest were used for implementing our method based on Deep learning, SVM, and Random forest, respectively. The following actual $R$ functions for them were called.

- res.dl <- h2o. deep learning $(x=1: 126, y=127$, training_frame $=$ train_data)

- res.svm <-svm(favoritebrand ., train_data)

- res.rf <- randomForest(favoritebrand .,train_data) For obtaining a recommended brand, the following function calls were used for the three methods, respectively.

- h2o.predict(object=res1.dl, newdata=test_data[,-127])

- $\quad \operatorname{predict}($ res.svm, newdata=test_data[1,-127])

- $\quad$ predict(res.rf, newdata=test_data, type="response")

\section{An Example of a Recommendation}

Assume that a user inputs her user profile where its gender is FEMALE, her height is $154 \mathrm{~cm}$ and her usual brands are "LOWRYS FARM", "Another Edition" and "mystic". With our recommendation method, her favorite brand was predicted and the result was "ZARA". Actually, the user wrote "ZARA" as her favorite brand in her profile. Although the correct favorite brand is obtained in this example, wrong prediction occurred.

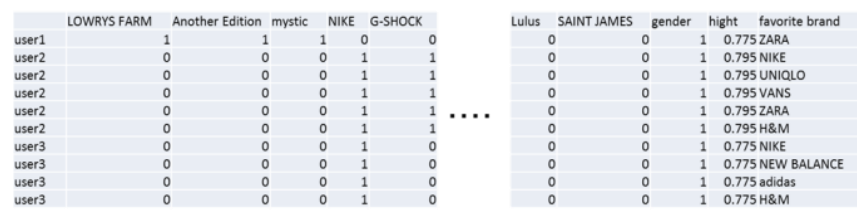

Fig. 3. Example of the data set.

\section{EXPERIMENTAL RESULTS}

TABLE IV: EVALUATION OF EXPERIMENTAL RESULTS

\begin{tabular}{cccc}
\hline \hline & DL & SVM & RF \\
\hline $\begin{array}{c}\text { Number of } \\
\text { Errors(\#Errortasks) } \\
\text { Error rate (\%) }\end{array}$ & 294 & 402 & 387 \\
$\begin{array}{c}\text { Number of correct } \\
\text { tasks (\#Correcttasks) }\end{array}$ & 125 & 95.9 & 92.4 \\
\hline \hline $\begin{array}{c}\text { Recommendation } \\
\text { Accuracy (\%) }\end{array}$ & $\underline{\mathbf{2 8 . 8}}$ & 4.1 & 32 \\
\hline \hline
\end{tabular}

Table IV shows the preliminary experiment results. We used Leave-one-out approach to calculate the accuracy. The accuracy used in the table is defined as the number of correct tasks divided by the number of task. In this case, since we conducted 419 task, the accuracy is \#Correcttasks / 419. The \#Correcttasks is 419-\#Errortasks

From the experimental results of the table, the accuracy of the deep learning was at least seven times higher than the SVM accuracy and three times or more higher than Random Forest (RF) accuracy. Deep learning was able to obtain a much higher accuracy than the other machine learning.

Since the number of different values in our "Favorite brands" item is 10 , the chance probability, which means the accuracy in the case of randomly selecting the favorite brand among ten brands without any smart method, is $10 \%$. The 
deep learning attained the higher accuracy than the value, although the accuracy of the other two methods is lower than the value. It is natural that the accuracy can be improved after tuning parameters in all three methods. The experiments after tuning are currently underway.

From this result, it was found that the deep learning can be applied to the fashion brand recommendation with high recommendation accuracy.

\section{RELATED WORK}

There are a few research studies of applying the deep learning for applications besides image or video recognition.

Huang et al. [6] proposed several new latent semantic models with deep architectures called DSSM. They used click-through data to optimize the parameters of their models by obtaining the correct document. The point is that they extended the linear semantic models to their non-linear model using multiple hidden representation layers as using deep learning. Their models lead to significant performance improvement on the document ranking task. Their target applications do not include information recommendation.

Unger et al. [7] suggested using high dimensional sensors, representing users' context for a CARS (Context-Aware Recommender System), in order to improve the quality of information gathered from many information sources that differ in the quality of information. Their proposed method can reduce the dimensionality by extracting latent contexts from data collected by mobile device sensors. They utilized unsupervised deep learning techniques on the collected data. Although their main application is the information recommendation, their system only uses the deep learning for selecting contexts such as light levels and locations to recommend objects depending on the obtained contexts.

Hao Wang et al. [8] proposed a new method for collaborative-filtering recommendation by using the deep learning, called Collaborative Deep Learning (CDL). In order to realize the CDL, they used and extended Bayesian based SDAE (Stacked Denoising AutoEncoders) model. It is unclear that their model can be applied in fashion-brand recommendation, because the number of favorite brands for each user is not large but small compared with that of their used data set and it is much hard for users to write and check their favorite-brand rating.

\section{CONCLUSION}

In this paper, we proposed a fashion-brand recommendation method that uses a deep learning technique. To create a learning model, three items, "gender," "height," and "brands user enjoys wearing," that were accurately entered were used. The preliminary experimental results show that our method outperforms SVM and RF, the conventional machine learning methods, from the recommendation accuracy viewpoint.

Our future work includes more detailed evaluation and improvement of the accuracy by extending the model in the deep learning.

\section{ACKNOWLEDGMENT}

This work was partially supported by MEXT-Supported Program for the Strategic Research Foundation at Private Universities 2015-2019.

\section{REFERENCES}

[1] Ministry of Economy Trade and industry Information Economy Division of the Commerce and Information Policy Bureau. (2016). Heisei 25(AD2013) Infrastructure Development Report in Accordance with the Japan Economic and Social Information Service. [Online]. Available:

http://www.meti.go.jp/meti_lib/report/2014fy/E004265.pdf

[2] M. Nakamura and Y. Kenichiro, “A study on the effects of consumer's personal difference on risk reduction behavior and internet shopping of clothes," Chukyo Business Review, vo1. 10, pp. 133-164, 2014.

[3] T. Kamishima, "Algorithms of recommender systems," Japanese Society for Artificial Intelligence, vol. 23, no. 1, pp. 89-103, 2008.

[4] Y. Wakita, K. Oku, H. H. Huang, and K. Kawagoe, "A fashion-brand recommender system using brand association rules and features," in Proc. 4th International Congress on Advanced Applied Informatics (AAI), pp. 719-720.

[5] A. Hideki, M. Yasuda, S. Maeda, and D. Okanohara, "Deep learning, kindaikagakusha in Japan," 2015.

[6] P. S. Huang, X. D. He, J. F. Gao, L. Deng, A. Acero, and L. Heck, "Latent contest-aware recommender systems," in Proc. ACM International Conference on Information and Knowledge Management (CIKM), 2013.

[7] M. Unger, "Latent context-aware recommender systems," ACM, pp. 383-386, 2015.

[8] H. Wang, N. Y. Wang, D. Y. Yeung, and M. Unger, "Collaborative deep learning for recommender systems," $A C M K D D^{\prime} 15$, pp. $1235-1244,2015$

Yuka Wakita is a graduate student at Ritsumeikan University. His research interest includes deep learning and recommender system.

Kenta Oku is a lecturer at Ritsumeikan University. His research interest includes recommender systems and web intelligence.

Kyoji Kawagoe is a professor at Ritsumeikan University. His research interest includes databases, information retrieval, and data science. 